\title{
Cardiac disease, cardiac rehabilitation and barriers to participation - an overview
}

\author{
Joan A Cebrick Grossman* \\ Department of Exercise Science and Sport, The University of Scranton, 100 Linden St., Scranton, PA 18510-4650, USA
}

\begin{abstract}
Cardiac disease is the primary cause of death in the United States and throughout the world. Patients who suffer from cardiac disease are most often diagnosed with vessel disease specific to the coronary arteries. Cardiac rehabilitation (CR) is a secondary prevention program designed to prevent the progression of this disease. A structured phase II CR program enhances the quality of life both physically and emotionally for patients with long-term outcomes; as such a program involves a comprehensive approach with the primary focus on the physical rehabilitation along with educational, psychological and social support. CR programs have shown promising results for improving clinical outcomes, yet participation remains disappointingly low. Barriers to participation have been identified as: gender, lack of physician referral, age, race/ethnicity and socioeconomic status. Recognizing and overcoming such barriers are necessary for the improvement of patient outcomes through increased CR enrollment and participation.
\end{abstract}

\section{Introduction}

Coronary artery disease, predominantly atherosclerotic in nature, is the most common form of cardiac disease and the primary cause of unanticipated death and disability for persons forty years of age and older [1]. Since the twentieth century, this disease remains the principal cause of death in this country and around the world $[2,3]$. The etiology of atherosclerosis is complex and multi-factorial. Progression of atherosclerosis may result in either a myocardial infarction (MI), better known as a "heart attack" and/or angina pectoris, characterized as recurring chest pain. A MI is the more severe of the two as it results in death of the myocardial tissue. Nearly all deaths from coronary heart disease are a result of a MI [4]. Angina pectoris is a result of myocardial ischemia due to reduced blood flow or increased demand for oxygen by the myocardium, which is reversible and does not lead to necrosis [5]. According to the American Academy of Cardiovascular and Pulmonary Rehabilitation, [6] cardiac rehabilitation (CR) is a comprehensive program that focuses primarily on the physical rehabilitation of a cardiac patient, yet also provides education, psychological and social support with a common goal of stabilizing and preventing the progression of this deadly disease. A structured CR program enhances the quality of life both physically and emotionally for patients with long-term outcomes [7-9]. Patients who typically experience a MI and/or coronary artery bypass surgery are those most referred to a CR program for care and recovery $[10,11]$. Throughout the past two decades, the empirical evidence supports that CR programs improve clinical outcomes for patients having suffered a MI including reducing mortality by $20-25 \%,[5,12,13]$. Despite such positive results, CR participation remains disappointingly low, with nearly one-third of eligible male participants and more than $80-89 \%$ of eligible female patients not participating in structured programs [12,14]. More recently it was reported that females were $36 \%$ less likely to participate than male counterparts [15]. Barriers to participation and program adherence have been identified as gender, physician referral, age, race/ ethnicity and socioeconomic status [16-18].

\section{Pathogenesis of atherosclerosis}

The term atherosclerosis is used interchangeably with the word arteriosclerosis although there are two different meanings. Arteriosclerosis is the more general term which means hardening of the arterial walls. Atherosclerosis is a disease process that leads to the development of fibrous, lipid-filled deposits, termed plaques or atheromas $[19,20]$. Overtime, the plaques can regress in nature or progress and become unstable possibly rupturing, resulting in a MI or stabilize and become hardened from calcium deposition [21].

Initially, it was believed that an injury to the endothelium resulted in the initiation of the atherosclerotic process [22]. Additional work by Ross [23] hypothesizes that some type of dysfunction rather than injury alone results in inflammation to the endothelium and initiates the atherosclerotic process. The exact basis of the dysfunction is unknown, though there are several speculated causes. The primary risk factors to endothelia inflammation include elevated and oxidized low-density lipoproteins (LDL), increased free radical exposure due to inhaled cigarette smoke, hypertension and diabetes mellitus. Other factors may include increased homocysteine levels, genetic changes, infectious microorganisms and/or a combination of these or other factors [21,23].

The precise mechanism of this dysfunction and inflammation is not clear, but what is understood are the types of cells involved in this process such as endothelial cells, smooth muscle, platelets, monocytes and lymphocytes [23]. According to Ross [23] and Ross and Glomset

Correspondence to: Joan A Cebrick Grossman, PhD, RD, Assistant Professor, Department of Exercise Science and Sport, The University of Scranton, 100 Linden St. Scranton, PA 18510-4650, USA, Tel: 570-941-4721; Fax: 570941-6209; E-mail: joan.grossman@scranton.edu

Key words: cardiac disease, cardiac rehabilitation, barriers to participation

Received: January 05, 2016; Accepted: January 20, 2016; Published: January 23, 2016 
[24], the theory of atheroma development is a result of damage to the endothelia cells wherein the underlying subendothelia connective tissue is exposed to LDL-cholesterol resulting in cellular damage. The body attempts to maintain homeostasis and repairs the damaged area through platelet aggregation and increased clotting around the injured site. If the injury and the response to it are limited, the lesion or atheroma becomes stabilized and the endothelium migrates into the lesion resulting in endothelia stabilization. However, if the injury is chronic as with hypertension and cigarette smoking, both of which are primary risk factors for disease, continual repair leads to smooth muscle proliferation, which results in vessel narrowing caused by the migration of smooth muscle into the area. The injured endothelium propagates subendothelia and monocyte attachment, which is believed to engulf cholesterol molecules. Foam cells develop due to an increase in LDL-cholesterol molecules and ultimately the infamous "fatty streak" results [24].

The "fatty streak" is the first designated lesion of atherosclerosis. It is believed to be present in all children by age ten, regardless of sex or race and has been identified in persons as young as eight months of age [25]. By age twenty-five, it is estimated that thirty percent of the aorta's intimal surface is covered with the "fatty streak" [21,24]. The "fatty streak" is clinically asymptotic, reversible in nature and is known to cause no significant vessel obstruction [25].

If given the opportunity to advance, the "fatty streak" develops into a more advanced lesion which becomes the "fibrous plaque." This second stage lesion represents advancing atherosclerosis. This "fibrous plaque" has a fibrous-like cover incorporating smooth muscles cells and cholesterol along with extracellular lipid and cellular debris [21,23,24].

The third and most advanced lesion in atherosclerosis is the "complicated lesion" and is appropriately termed arteriosclerosis. This lesion is far different than the "fibrous plaque," such that, it is hardened as a result of calcification and hemorrhage, thrombosis and cellular necrosis often results. It is this lesion which is associated with ischemia and the occlusion of blood. Overtime, the "complicated lesion" protrudes into the arterial lumen and decreases blood flow, impairing arterial vessel dilation $[23,24]$.

\section{Cardiovascular disease diagnoses and procedures}

As blood flow to the heart muscle becomes inadequate, consequences, such as a MI or angina pectoris, may result. Myocardial infarction, more commonly referred to as coronary occlusion, coronary thrombosis, or in lay terms, a heart attack, is the more severe of the two, as death to the myocardial tissue results. Depending upon the size and the location of the infarct, death to an individual is often the consequence [5].

Angina pectoris, understood as chest pain, is also a result of myocardial ischemia, but does not lead to necrosis. It is classified as stable and unstable. Stable angina is stimulated by factors that increase the demand for oxygen to the heart tissue. Some examples include exercise, smoking, high altitude and anxiety. Unstable angina is far more dangerous since it is triggered with minimal exertion, even at rest. Percutaneous coronary interventions (PCIs) consist of a variety of procedures which treat cardiac patients experiencing ischemic processes such as a MI or angina pectoris $[19,20]$. A common PCI is a percutaneous transluminal coronary angioplasty (PTCA), better understood as "the balloon" procedure and is often referred to as a "plain old" balloon angioplasty (POBA). This procedure involves a balloontipped catheter that is apposition to the atheroma within the coronary artery. Inflation of the balloon compresses the lesion or plaque against the side of the arterial wall re-establishing the lumen with resumption of blood flow. The procedure is often accompanied by the placement of a coronary stent, which is an "expandable wire-mesh tube" that works to splint the lumen open to maintain lumen size [19,20]. Drug-eluding stents work to keep the vessel lumen open, slowly releasing medication into the vessel wall to prevent a complication known as re-stenosis or re-narrowing as a result of scar tissue development from the stent $[19,20]$.

There are circumstances that do not allow for PCIs, such as those patients who have numerous blockages throughout the coronary vessels. This is understood as a "vascular tree," meaning the branching of the vessels similar to that of a tree have blockages in many sites and stent placement is not an option. In such cases, coronary artery bypass graft more commonly known as CABG, pronounced "cabbage" is typically recommended. This is the most common form of major heart surgery performed in the United States and in Western countries [26]. This surgical procedure involves supplying vessels from other parts of the body, such as the internal mammary artery from within the chest wall, the saphenous vein from the leg or the radial vein from the forearm. These vessels are attached above and below the occluded coronary arteries and literally bypass them to allow for increased blood flow to the heart muscle [26]. Depending upon the number of occluded vessels, one may have a single, double, triple or quadruple bypass, indicating the number of obstructions needing the blood flow bypass. The number of bypasses is not indicative of the level of disability, as it is more of a reflection of those lesions that require bypass. For example, a patient who undergoes a single bypass of a critical lesion may have more ischemic disease than a patient who had a triple bypass surgical procedure. However, the single bypass patient may not have had good vessels by which to bypass as the vessels may have been too small and/ or too calcified with no area free of ischemic disease or the vessels may have been deep within the cardiac tissue and not on the surface where the bypass surgery is performed.

Patients who experience a MI and/or CABG, have been most commonly referred to a CR program [10,5]. However, in March 2006 and February 2014, the Center for Medicare and Medicaid Services (CMS), the federal agency that determines clinical diagnoses appropriate for Medicare and Medicaid reimbursement, broadened the diagnoses for eligible CR coverage. These additional diagnoses initially included stable angina pectoris, heart valve repair or replacement, PTCA or coronary stenting, heart and heart/lung transplant and more recently included chronic heart failure (CHF) [10]. The expansion of these diagnostic eligibility pools supported the need for $\mathrm{CR}$ as a necessity for patient care and recovery.

\section{Cardiac rehabilitation}

According to the American Academy of Cardiovascular and Pulmonary Rehabilitation, [6], CR is a secondary prevention program, meaning disease prevention is secondary to treatment, which is primary. $\mathrm{CR}$ is a multi-faceted, interdisciplinary program that focuses on the physical rehabilitation of the patient and also includes educational, psychological and social support, with a common goal of stabilizing and preventing disease progression. In addition, CR is considered a Class 1 indication, meaning it is useful and effective [27], as it is a comprehensive program, defined by four phases, all of which involve the progression of the patient, though treatment and rehabilitation specific to the cardiovascular system.

Phase I is care provided in a hospital, ordered by either a primary 
care physician or a cardiologist as a result of a cardiac event. The length of stay for phase I is typically three to five days post-event depending upon patient diagnosis and progress [5,7]. The primary goal of phase I $\mathrm{CR}$ is patient mobilization and weaning the patient from any dependent oxygen [28]. This physical rehabilitation is critical to patient success as patients receiving daily $\mathrm{CR}$ through ambulation, recover quicker and return home sooner as opposed to the time when CR was not an integral part of the continuum of care for such patients [29].

The patient status for phases II through IV CR is that of an outpatient, however the latter three phases may be located either within a hospital or an outpatient clinic, depending upon the program. Phase II CR intimately rehabilitates the patient through physical exercise and patient education which requires a physician referral from either a cardiologist or a primary care physician [10]. Phase II is typically twelve weeks in length with the patient attending three exercise sessions each week, for a total of thirty-six sessions. A typical class session includes a 5-10 minute warm-up, an average exercise session of 45 minutes while alternating upper and lower body exercises along with a final 5-10 minute cool-down segment [28]. Blood pressure is monitored and recorded with pre- and post-exercise measurements, along with several measurements taken throughout the exercise session. Exercise intensities which are diagnoses dependent, are calculated using agepredicted maximum heart rate, heart rate reserve or $+20-30$ beats above resting heart rate [28]. The perceived exertion scale [30], more commonly known as rate of perceived exertion or RPE is used to subjectively determine intensity. Exercise prescriptions are modified based upon other medical problems such as peripheral vascular disease, diabetes and arthritis. Exercise tolerance is closely monitored during each exercise session with a three-lead electrocardiogram (ECG) recorded connection. A cool-down segment is offered at the conclusion of the exercise bout which supports and educates patients regarding stress reduction and relaxation.

In addition to the exercise sessions, patient education is an important component of a phase II CR program. Some examples of educational topics include nutrition, medications, stress reduction, risk factor reduction such as smoking cessation and the importance of exercise. Patient education pertaining to lifestyle changes is necessary to not only treat, but to prevent the progression of the disease. Some CR programs offer educational sessions while others provide educational materials or a combination of both. It is highly encouraged, that all patients and primary caregivers attend the educational sessions offered and/or read the educational materials provided to better understand the successful management of this disease.

Phase III CR is achieved once a patient has completed the prior two phases. This phase is self-referred, requiring a physician's consent but not a physician referral. Phase III CR involves independent exercise with sporadic ECG monitoring such as once a month for a three-month period and is self-paid with no insurance reimbursement [31]. Phase IV CR is defined as independent exercise, free from ECG monitoring and/or blood pressure measurements, requires physician consent, not a referral, and is self-paid. Independent exercise for any CR patient is highly recommended to ultimately prevent the progression of the disease [32].

It is well documented that a structured CR program enhances the quality of life for patients, as it improves clinical outcomes for patients reducing all-cause and cardiac mortality, nonfatal reinfarction and reduced hospitalization rates [27]. Despite such positive results, program participation remains disappointingly low, with only $25-31 \%$ of eligible male participants and only $11-20 \%$ eligible female patients participating in structured programs $[12,14]$. And, more recently it was reported that females were $36 \%$ less likely to participate than male counterparts [15].

\section{Barriers to cardiac rehabilitation}

The improvement of patient outcomes should remain at the forefront of patient care, however, all too often, patients are precluded from the care needed for complete and full recovery. In particular, CR is an expected outcome for all cardiac patients, yet multiple barriers have been identified that limit patient participation, specific to phase II CR. Such barriers to participation and program adherence include: gender, lack of physician referral, age, race/ethnicity and socioeconomic status $[12,14,15,17,18]$. These same barriers have been identified as primary health disparities in the Healthy People 2020 report [16].

\section{Gender}

Heart disease surpasses cancer as the principal cause of death for women in the United States with nearly 300,000 victims each year [11]. More than one in three women over the age of 25 die of either a heart attack or stroke each year, as opposed to breast cancer which accounts for one in every thirty deaths $[19,20]$. The need for quality patient care is essential for women with cardiac disease. However, the data supports just the opposite, wherein women are not only underrepresented in CR programs, they are not referred [17,34], as cardiac disease is misunderstood as a man's disease. A recent metaanalysis looked specifically at the sex differences in CR enrollment [15], with the understanding that women who receive CR treatment display positive health outcomes, in particular decreased cardiac mortality [15], yet women were $36 \%$ less likely to enroll than men.

\section{Lack of physician referral}

The lack of physician referral particularly for women has been identified as an access barrier to outpatient, phase II CR programs and without the required physician referral, participation simply is not possible $[18,33,34]$. Along with the lack of physician referral specific to a women's centered CR program, Rolfe et al. [35] also identified transportation as an issue. Such that not only is the lack of physician referral a barrier to CR enrollment, the inability to get to the program is yet another barrier which prohibits participation, the lack of transportation has also been cited in a previous study specific to CR participation barriers [36]. Communication between the patient and physician is a necessary tool which may help to overcome some of these barriers. This was recently reported in one study which focused on strategies to improve the dialogue between patients and physicians for improved chronic disease management [37]. There is a need to educate physicians regarding the prevalence of cardiac disease among women along with the understanding of the improved patient outcomes for enhanced care and recovery.

\section{Age}

The proportion of elderly individuals within our population has steadily increased over the past several decades, as have those with coronary disease [14]. According to Clark et al. [38], the elderly are not referred to CR for care as compared to younger patients. Even in the very old, those 75 years and older, participation was lower as they were not referred as were younger patients, however the improvement in physical function is similar to that of younger patients [39]. Evidence suggests the lack of use of CR services is in part, due to physician preconceptions about less program value in older patients while clinical 
evidence supports that CR services are of significant value and worth to patients regardless of age [14]. Another study concurred that older patients endorsed barriers to CR which included: already exercising at home, misperceptions of exercise as painful, or not improving one's health, lack of physician referral and simply not knowing about the program [40,41].

\section{Race and socioeconomic class}

Race and socioeconomic class have been indicated as a social barriers to participation in an outpatient, phase II CR program $[42,43]$. Whites were more likely to participate than ethnic minorities and minorities reported greater financial barriers to participation [42]. Women of color with lower income levels were less likely referred to a phase II CR program, than white females [43]. Additionally, minority women had a worse prognosis following hospitalization indicating a greater need for outpatient, phase II services for such patients. There have been other supportive studies identifying ethnic minorities as less referred to CR services [43-45]. Several other studies identified that individuals of a lower socioeconomic level are simply not referred for outpatient CR services $[42,44,45]$. Such patients are in need of care but simply are not receiving the care which is imperative to patient success and recovery. The decreased utilization of such services may lead to further disparity in cardiac outcomes which presents an ethical dilemma regarding patient care, specific points indicated in Healthy People 2020 [16]. The literature clearly supports the understanding that numerous barriers prevent one from enrolling into a CR program prohibiting patient care. Future research should focus on overcoming such barriers in order to provide the best possible care for patients.

\section{Future research}

Despite the multi-faceted benefits to CR, program, participation remains low. Alternative options to a traditional program offering might be beneficial to increase patient enrollment and participation. For the past two decades, such options have been examined and have included a more personalized approach with the hopes of meeting individual needs [46], while others have suggested programs designed through different channels such as home-based, community or hospital-based programs that fit one's lifestyle [47]. Carlson et al. [48], designed a reduced-cost modified protocol for patients to promote independent exercise. Patients exercised for one month as in a traditional program. The change in protocol occurred in week five, wherein patients were directed to exercise independently at a local facility, three times each week for the following six months. It was concluded that such a program was effective in maintaining exercise along with improved physiological measurements as compared to a traditional 12-week program. However, the subjects in this particular study were identified as low- to moderate-risk patients and all too often cardiac rehab patients are high-risk patients.

Although such a modified program may work as an alternative for some, it would not fit the needs of those patients with the greatest risks. More recent work has suggested home-based models of care for patients based upon patient risk [49], while others have suggested internet- and mobile-based lifestyle interventions as possible alternatives particularly for those patients with the lowest participation rates, such as those in lower socioeconomic class and racial/ethnic minorities [50].

\section{Conclusion}

Participation of patients eligible for CR remains disappointingly low even with the acknowledgment and expansion of diagnoses for myocardial infarctions and chronic heart failure through CMS in 2006 and 2014, respectively. The identification of both access and social barriers which have been identified as limiting enrollment and participation is an important step in improving patient outcomes. Much work is needed to effectively increase the enrollment and participation rates of these patients either through the altering of the classic structured phase II CR program or via new and revised programs that will meet the needs of these patients in the $21^{\text {st }}$ century.

\section{Declaration of conflicting interests}

The author(s) declared no potential conflicts of interest with respect to research, authorship, and/or publication of this article.

\section{Funding}

No financial funding was received for this study.

\section{References}

1. Menotti A, Kromhout D, Blackburn H, Jacobs D, Lanti M (2004) Forty-year mortality from cardiovascular diseases and all causes of death in the US Railroad cohort of the Seven Countries Study. Eur J Epidemiol 19: 417-424. [Crossref]

2. Gibbons RJ, Jones DW, Gardner TJ, Goldstein LB, Moller JH, et al. (2008) The American Heart Association's 2008 Statement of Principles for Healthcare Reform. Circulation 118: 2209-2218. [Crossref]

3. Gillum RF, Mussolino ME, Madans JH (2004) Relation between region of residence in the United States and hypertension incidence: The NHANES I epidemiologic followup study. Journal of the National Medical Association 96: 625-651. [Crossref]

4. Wyer SJ, Earll L (2001) Predicting attendance at cardiac rehabilitation: A review and recommendations. Coronary Health Care 5: 171-17.

5. Leon AS, Franklin BA, Costa F, Balady GJ, Berra KA, et al. (2005) Cardiac rehabilitation and secondary prevention of coronary heart disease: An American Hear Association scientific statement from the council on clinical cardiology and the council on nutrition, physical activity and metabolism in collaboration with the American Association of Cardiovascular and Pulmonary Rehabilitation. Circulation 111: 369376. [Crossref]

6. American Association of Cardiovascular and Pulmonary Rehabilitation (AACVPR) Fast facts: Referral/resource pages for cardiac rehabilitation.

7. Lawler PR, Filion KB, Eisenberg MJ (2011) Efficacy of exercise-based cardiac rehabilitation post-myocardial infarction: A systematic review and meta-analysis of randomized controlled trials. American Heart Journal 162: 571-584. [Crossref]

8. Hammill BG, Curtis LH, Schulman KA, Whellan DJ (2010) Relationship between cardiac rehabilitation and long-term risks of death and myocardial infarction among elderly Medicare beneficiaries. Circulation 5: 63-70. [Crossref]

9. Jackson L, Leclerc J, Erskine Y, Linden W (2005) Getting the most out of cardiac rehabilitation: a review of referral and adherence predictors. Heart 91: 10-14. [Crossref]

10. Centers for Medicare and Medicaid Services (CMS) (2014) Decision memo for cardiac rehabilitation programs (CAG-00089R).

11. Centers for Medicare \& Medicaid Services (CMS), HHS (2009) Medicare program changes to the hospital outpatient prospective payment system and CY 2010 payment rates; changes to the ambulatory surgical center payment system and CY 2010 payment rates. Final rule with comment period. Fed Regist 74: 60315-60983. [Crossref]

12. Barber K, Stommel M, Kroll J, Holmes-Rovner M, McIntosh B (2001) Cardiac rehabilitation for community-based patients with myocardial infarction: Factors predicting discharge recommendation and participation. Journal of Clinical Epidemiology 54: 1025-1030. [Crossref]

13. Lau J, Antman EM, Jimenez-Silva J, Kupelnick B, Mosteller F, et al. (1992) Cumulative meta-analysis of therapeutic trials for myocardial infarction. N Engl J Med 327: 248254. [Crossref]

14. Suaya JA, Shepard DS, Normand SL, Ades PA, Prottas J, et al. (2007) Use of cardiac rehabilitation by Medicare beneficiaries after myocardial infarction or coronary bypass surgery. Circulation 116: 1653-1662. [Crossref]

15. Samaoya L, Grace SL, Gravely S, Benz Scott L, Marzolini S, et al. (2013) Sex differnces in cardiac rehabilitation enrollment: a meta-analysis. Canadian Journal of Cardiology. 
16. United States Department of Health and Human Services, Office of Minority Health (2010) National Partnership for Action to End Health Disparities. The National Plan for Action.

17. Sanderson BK, Bittner V (2005) Women in cardiac rehabilitation: outcomes and identifying risk for dropout. Am Heart $J$ 150: 1052-1058. [Crossref]

18. Evenson KR, Fleury J (2000) Barriers to outpatient cardiac rehabilitation participation and adherence. J CardiopulmRehabil 20: 241-246. [Crossref]

19. American Heart Association (AHA) (2014a) Angina pectoris, atherosclerosis, myocardial infarction.

20. American Heart Association (AHA) (2014b) Women and cardiovascular disease statistics.

21. Ross R (1986) The pathogenesis of atherosclerosis--an update. N Engl J Med 314 488-500. [Crossref]

22. Ross R, Glomset JA (1973) Atherosclerosis and the arterial smooth muscle cell: Proliferation of smooth muscle is a key event in the genesis of the lesions of atherosclerosis. Science 180: 1332-1339. [Crossref]

23. Ross R (1999) Atherosclerosis--an inflammatory disease. N Engl J Med 340: 115-126. [Crossref]

24. Ross R, Glomset JA (1976) The pathogenesis of atherosclerosis (first of two parts). $N$ Engl J Med 295: 369-377. [Crossref]

25. Stary HC (1989) Evolution and progression of atherosclerotic lesions in coronary arteries of children and young adults. Arteriosclerosis 9: I19-32. [Crossref]

26. SundtTM (2014) What is coronary artery bypass grafting (CABG)? The Society of Thoracic Surgeons.

27. Oldridge N (2012) Exercise-based cardiac rehabilitation in patients with coronary heart disease: meta-analysis outcomes revisited. Future Cardiol 8: 729-751. [Crossref]

28. American College of Sports Medicine (ACSM) (2014) Guidelines for Exercise Testing and Prescription, 9th Edition, Philadelphia, PA: Wolters Kluwer, Lippincott, Williams\& Wilkins.

29. Clark EI, Roberts CL, Traylor KC (2004) Cardiovascular single-unit stay: a case study in change. Am J Crit Care 13: 406-409. [Crossref]

30. Borg GAV (1962) Physical performance and perceived exertion. Lund, Sweden: Gleerup.

31. Mampuya WM (2012) Cardiac rehabilitation past, present and future: an overview. CardiovascDiagnTher 2: 38-49. [Crossref]

32. Hughes AR, Gillies F, Kirk AF, Mutrie N, Hillis WS, et al. (2002) Exercise consultation improves short-term adherence to exercise during phase IV cardiac rehabilitation: a randomized, controlled trial. Journal of Cardiopulmonary Rehabilitation 22: 421-425.

33. Dalal H, Evans PH, Campbell JL (2004) Recent developments in secondary prevention and cardiac rehabilitation after acute myocardial infarction. British Medical Journal 328: 693-697. [Crossref]

34. Plach SK (2002) Women and cardiac rehabilitation after heart surgery: Patterns of referral and adherence. Rehabilitation Nursing 27: 104-109. [Crossref]

35. Rolfe DE, Sutton EJ, Landry M, Sternberg L, Price JA (2010) Women's experiences accessing a women-centered cardiac rehabilitation program: a qualitative study Journal of Cardiovascular Nursing 25: 332-41. [Crossref]

36. Daly J, Sindone AP, Thompson DR, Hancock K, Chang E, et al. (2002) Barriers to participation in and adherence to cardiac rehabilitation programs: A critical literature review. Progress in Cardiovascular Nursing 17: 8-17. [Crossref]

37. Sakar U, Schilliner D, Bibbins-Domingo K, Napoles A, Karliner 1, et al. (2011) Patientphysicians' information exchanges in outpatient cardiac care: time for a heart to heart? Patient Education and Counseling 85: 173-9. [Crossref]

38. Clark AM, Sharp C, MacIntyre PD (2002) The role of age in moderating access to cardiac rehabilitation in Scotland. Ageing and Society 22: 501-515.

39. Audelin MC, Savage PD, Ades PA (2008) Exercise-based cardiac rehabilitation for very old patients ( $>$ or $=75$ years): focus on physical function. $J$ CardiopulmRehabilPrev 28 : 163-173. [Crossref]

40. Grace SL, Shanmugasegaram S, Gravely-White S, Brual J, Suskin N, et al. (2009) Barriers to cardiac rehabilitation: does age make a difference? Journal Cardiopulmonary Rehabilitation and Prevention 29: 183-187. [Crossref]

41. Grace SL, Angevaare KL, Reid RD, Oh P, Anand S, et al. (2012) Effectiveness of inpatient and outpatient strategies in increasing referral and utilization of cardiac rehabilitation: a prospective, multi-site study. Implement Sci 7: 120. [Crossref]

42. Mochari H, Lee JW, Kligfield P, Mosca L (2006) Ethnic differences in barriers an referral to cardiac rehabilitation among women hospitalized with coronary heart disease. Preventive Cardiology 9: 8-13. [Crossref]

43. Allen JK, Scott LB, Stewart KJ, Young DR (2004) Disparities in women's referra to and enrollment in outpatient cardiac rehabilitation. J Gen Intern Med 19: 747-753. [Crossref]

44. Gregory PC, LaVeist TA, Simpson C (2006) Racial disparities in access to cardiac rehabilitation. Am J Phys Med Rehabil 85: 705-710. [Crossref]

45. Jolly K, Lip GY, Taylor RS, Mant JW, Lane DA, et al. (2005) Recruitment of ethnic minority patients to a cardiac rehabilitation trial: The Birmingham rehabilitation uptake maximization (BRUM) study [ISRCTN72884263]. BioMed Central Medical Research Methodology 5: 1-8.

46. Oldridge N (1991) Compliance with cardiac rehabilitation services. Journal of Cardiopulmonary Rehabilitation 11: 115-127.

47. Thompson D, Bowman G, Kitson A, de Bono D, Hopkins A (1997) Cardiac rehabilitation services in England and Wales: A national survey. International Journal of Cardiology 59: 299-304.

48. Carlson JJ, Johnson JA, Franklin BA, VanderLaan RL (2000) Program participation, exercise adherence, cardiovascular outcomes, and program cost of traditional versus modified cardiac rehabilitation. American Journal of Cardiology 86: 17-23. [Crossref]

49. Brual J, Gravely S, Suskin N, Stewart DE, Grace SL (2012) The role of clinical and geographic factors in the use of hospital versus home-based cardiac rehabilitation. Int $J$ Rehabil Res 35: 220-226. [Crossref]

50. Brewer LC, Kaihoi B, Zarling KK, Squires RW, Thomas R, et al. (2015) The use of virtual world-based cardiac rehabilitation to encourage health lifestyle choices among cardiac patients: intervention development and pilot study protocol. Journal of Medical Internet Research, Research Protocols 8: e39. [Crossref]

Copyright: (C2016 Grossman J. This is an open-access article distributed under the terms of the Creative Commons Attribution License, which permits unrestricted use, distribution, and reproduction in any medium, provided the original author and source are credited. 